ISSN 0103-9954

\title{
RELAÇÕES FLORÍSTICAS E FITOSSOCIOLOGIA DE UMA FLORESTA OMBRÓFILA MISTA MONTANA SECUNDÁRIA EM LAGES, SANTA CATARINA
}

\author{
FLORISTICS RELATIONS AND PHYTOSOCIOLOGY OF A SECONDARY MONTANE \\ ARAUCARIA FOREST FRAGMENT IN LAGES, SANTA CATARINA STATE
}

\author{
Ana Carolina da Silva ${ }^{1}$ Pedro Higuchi ${ }^{2}$ Manoela Drews de Aguiar ${ }^{3}$ \\ Marcelo Negrini ${ }^{4}$ João Fert Neto ${ }^{5}$ André Felipe Hess ${ }^{6}$
}

\begin{abstract}
RESUMO
O presente estudo teve como objetivos caracterizar a estrutura fitossociológica de um fragmento de floresta secundária em Lages, SC, contextualizar a composição florística do componente arbóreo do fragmento em relação a outros remanescentes da região do Planalto Sul-Catarinense e avaliar a influência de variáveis geográficas e climáticas sobre os padrões observados. O levantamento da composição florística e estrutural da vegetação arbórea, do fragmento estudado, foi conduzido em 25 parcelas de $400 \mathrm{~m}^{2}$, onde todos os indivíduos arbóreos com DAP (diâmetro medido à altura do peito) $\geq 5 \mathrm{~cm}$ foram medidos e identificados. Para o estudo das relações florísticas entre o fragmento estudado e outros remanescentes da região foi utilizada a Árvore de Regressão Multivariada, que utilizou matrizes florísticas de espécies arbóreas de presença e ausência e de variáveis geográficas e climáticas de cada remanescente. Foram encontradas 87 espécies, distribuídas em 36 famílias botânicas. As espécies de maior valor de importância no fragmento estudado foram Araucaria angustifolia (Bertol.) Kuntze e Podocarpus lambertii Klotzsch. A distribuição diamétrica da comunidade demonstrou padrão próximo ao J invertido e as populações tiveram padrões de distribuição diferenciados. Foram descriminados três agrupamentos florísticos associados à sazonalidade térmica e à temperatura média anual. O presente fragmento esteve agrupado a outras áreas de sazonalidade térmica maior ou igual a $3,053^{\circ} \mathrm{C}$.
\end{abstract}

Palavras-chave: Árvore de Regressão Multivariada; estrutura; Floresta de Araucária.

\section{ABSTRACT}

This study investigated the floristic composition and the tree community structure in a secondary Araucaria Forest fragment in the Southern Plateau Region of Santa Catarina state, Brazil, the floristic context of this fragment in relation to other areas of Araucaria Forest in the region and the influence of climatic and

1. Engenheira Florestal, Dr $^{\mathrm{a}}$., Professora Adjunto do Departamento de Engenharia Florestal, Universidade do Estado de Santa Catarina, Av. Luiz de Camões, 2090, Bairro Conta Dinheiro, CEP 88520-000, Lages (SC). carol_sil4@yahoo.com.br

2. Engenheiro Florestal, Dr., Professor Adjunto do Departamento de Engenharia Florestal, Universidade do Estado de Santa Catarina, Av. Luiz de Camões, 2090, Bairro Conta Dinheiro, CEP 88520-000, Lages (SC). higuchip@gmail.com

3. Estudande, Curso de Engenheira Florestal, Departamento de Engenharia Florestal, Universidade do Estado de Santa Catarina, Av. Luiz de Camões, 2090, Bairro Conta Dinheiro, CEP 88520-000, Lages (SC). manoo aguiar@hotmail.com

4. Estudande, Curso de Engenheira Florestal, Departamento de Engenharia Florestal, Universidade do Estado de Santa Catarina, Av. Luiz de Camões, 2090, Bairro Conta Dinheiro, CEP 88520-000, Lages (SC). engfmarcelonegrini@hotmail.com

5. Engenheiro Florestal, Dr., Professor Adjunto do Departamento de Engenharia Florestal, Universidade do Estado de Santa Catarina, Av. Luiz de Camões, 2090, Bairro Conta Dinheiro, CEP 88520-000, Lages (SC). fert@cav.udesc.br

6. Engenheiro Florestal, Dr., Professor Adjunto do Departamento de Engenharia Florestal, Universidade do Estado de Santa Catarina, Av. Luiz de Camões, 2090, Bairro Conta Dinheiro, CEP 88520-000, Lages (SC). hess@cav.udesc.br

Recebido para publicação em 26/10/2009 e aceito em 17/12/2010 
geographic variables on the clustering patterns observed. The phytosociological survey of tree vegetation was assessed through $25,400 \mathrm{~m}^{2}$, plots, where all trees with $\mathrm{DBH}$ (diameter at breast height) $\geq 5 \mathrm{~cm}$ were measured and identified.The floristic relationship among remnants and the influence of climatic and geographic variables were analized through a Multivariate Regression Tree, using a presence-absence species matrix and a climatic-geographic variables matrix. In the study area, 87 species were found, distributed into 36 botanic families. The species with the highest importance value were Araucaria angustifolia (Bertol.) Kuntze and Podocarpus lambertii Klotzsch. While the distribution of individuals in diametric classes was similar to an inverted $\mathrm{J}$ shape for the community, the populations demonstrated different shapes of diametric distribution. In the region, three floristic clusters were discriminated, influenced mainly by temperature seasonality and annual mean temperature. The study site was grouped with together with other areas presenting temperature seasonality higher or equal to $3.053^{\circ} \mathrm{C}$.

Keywords: Multivariate Regression Tree; structure; Araucaria Forestry.

\section{INTRODUÇÃO}

AFloresta Ombrófila Mista foi intensamente explorada no sul do Brasil sendo que, em Santa Catarina, essa exploração ocorreu especialmente durante as décadas de 50 e 60 durante o "Ciclo da Araucária". Como resultado, houve grande fragmentação de habitat, restando remanescentes florestais com diferentes estados de conservação decorrentes, na maioria das vezes, de cortes seletivos. Os remanescentes impactados por cortes seletivos ou corte raso podem ser considerados florestas secundárias de acordo com a Resolução n. 10 do CONAMA de 1993, que define a vegetação secundária como a "resultante dos processos naturais de sucessão, após supressão total ou parcial da vegetação primária por ações antrópicas ou causas naturais, podendo ocorrer árvores remanescentes da vegetação primária”. As florestas secundárias também podem ser definidas como aquelas formadas após o corte raso da vegetação primária (GREIGSMITH, 1952; FINEGAN, 1992) ou aquelas formadas após algum tipo de perturbação humana (BROWN e LUGO, 1990). Para Corlett (1994), são consideradas florestas secundárias àquelas formadas após a interrupção de sua continuidade, levando a modificações estruturais e, ou, florísticas.

Apesar de serem florestas alteradas, essas florestas possuem elevada importância ecológica por apresentarem, segundo Pereira e Vieira (2001), rápido crescimento e acúmulo de carbono atmosférico, controle da erosão, conservação de nutrientes, benefícios hidrológicos e manutenção da biodiversidade residual e de migrantes. Além disso, funcionam como stepping-stones, ou corredores florestais, interligando áreas. Por isso, estudos que objetivem conhecer sua florística e estrutura, assim como analisar suas relações florísticas, podem servir de subsídios para planos de conservação de remanescentes florestais e recuperação de áreas degradadas.

A área do presente estudo é uma floresta secundária que sofreu corte seletivo no passado, principalmente de Araucaria angustifolia e, atualmente, tem seu sub-bosque roçado pelos proprietários das terras, especialmente na borda da floresta. Esta prática é comum na região e tem o intuito de suprimir a regeneração natural para facilitar a entrada e permanência do gado dentro da floresta, pois esta o protege contra o frio intenso da região, com ocorrência frequente de geadas e esporádica de neve no inverno. Em Santa Catarina existem poucos estudos em remanescentes de Floresta Ombrófila Mista, sendo que, no Planalto Catarinense, destacam-se os estudos de Falkenberg (2003) no Morro da Igreja, Serra do Corvo e Serra do Rio do Rastro, Formento et al. (2004) em Campo Belo do Sul; Eskuche (2007) em Bom Jardim da Serra; e Higuchi et al. (dados não publicados) em Painel, Lages e Campos Novos. Assim, os objetivos deste trabalho foram: I) caracterizar a estrutura fitossociológica de um fragmento de Floresta Ombrófila Mista Montana secundária, em Lages, SC; II) contextualizar a composição florística do componente arbóreo do fragmento em relação a outros remanescentes da região do Planalto SulCatarinense; e III) avaliar a influência de variáveis espaciais geográficas e climáticas sobre os padrões observados.

\section{MATERIAL E MÉTODO}

O estudo foi realizado em um fragmento de floresta secundária de 22 ha no município de Lages, $\mathrm{SC}$, na região do Planalto Sul-Catarinense (Figura 1). Lages está localizado na latitude $27^{\circ} 48^{\prime} 58^{\prime}$ 'S e

Ci. Fl., v. 22, n. 1, jan.-mar., 2012 


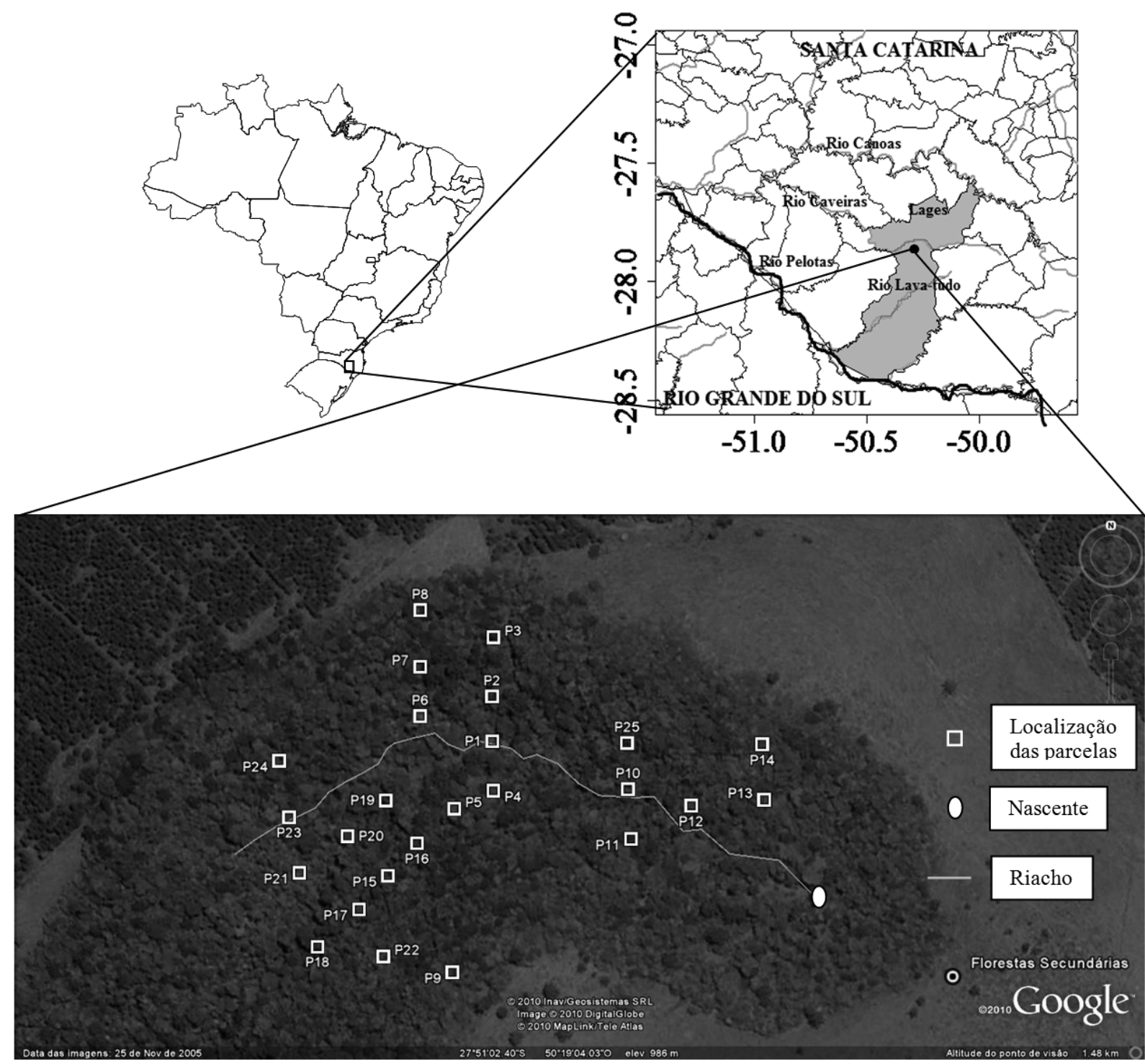

FIGURA 1: Fragmento de Floresta Ombrófila Mista Montana estudado no município de Lages, Santa Catarina, e a localização das parcelas para o levantamento fitossociológico.

FIGURE 1: Montane Araucaria Forest fragment studied and plot locations for the phytosociological survey in the municipality of Lages, Santa Catarina.

longitude $50^{\circ} 19^{\prime} 30^{\prime \prime} \mathrm{O}$, e possui altitude em torno de $916 \mathrm{~m}$. O clima predominante é Cbf, de acordo com a classificação de Köppen, a precipitação anual média é de $1.479,48 \mathrm{~mm}$, bem distribuída no ano, e a temperatura anual média é de $16^{\circ} \mathrm{C}$ (BRASIL, 1992). O município está inserido na Bacia Hidrográfica do Rio Canoas e do Rio Pelotas, com topografia de suave-ondulada a ondulada, sendo a vegetação classificada como Floresta Ombrófila Mista Montana (IBGE, 1992). O fragmento possui grande heterogeneidade ambiental, com a presença de uma nascente, de um riacho e de variações topográficas, com áreas de baixada, por onde passa o curso de água, encostas íngremes, grotas e topos de morros. Os tipos de solos predominantes são Cambissolos nas baixadas e Neossolos Litólicos nas encostas.

O levantamento da composição florística e estrutural da vegetação arbórea foi conduzido em 25 parcelas de $400 \mathrm{~m}^{2}(20 \times 20 \mathrm{~m})$ (Figura 1), totalizando 1 ha amostrado. As parcelas foram distribuídas de forma estratificada-sistemática em todo o fragmento, distanciadas $20 \mathrm{~m}$ entre si em cada estrato, de forma a procurar amostrar adequadamente as suas variações ambientais, tais como borda $\mathrm{x}$ interior, variações topográficas e proximidade do curso de água. 
Nelas, foram medidos (DAP e altura), coletados e identificados todos os indivíduos arbóreos vivos que apresentaram DAP (diâmetro à altura do peito a $1,30 \mathrm{~m}) \geq 5 \mathrm{~cm}$. Indivíduos com troncos múltiplos foram medidos quando a raiz da soma dos quadrados dos DAPs foi maior ou igual a $5,0 \mathrm{~cm}$. Para complementar a lista florística, também foram feitos caminhamentos no fragmento e identificação de espécies arbóreas, não encontradas nas parcelas. As identificações foram realizadas por meio de literatura e consulta a especialistas. As espécies foram classificadas nas famílias de acordo com o sistema APG III (ANGIOSPERM PHYLOGENY GROUP, 2009) e depositadas no Herbário LUSC na Universidade do Estado de Santa Catarina. A composição florística foi analisada pelo estimador de riqueza Jackknife de primeira e segunda ordem (HELTSHE e FORRESTER 1983) e a diversidade foi avaliada pelo índice de Shannon-Wiener (H') e equabilidade de Pielou (J') (BROWER e ZAR 1984), calculados pelo programa $R$ (R Development Core Team, 2009).

A estrutura do componente arbóreo foi descrita a partir do cálculo, para cada espécie, dos parâmetros quantitativos (MUELLER-DOMBOIS e ELLENBERG, 1974): densidade absoluta e relativa, frequência absoluta e relativa, dominância absoluta e relativa e valor de importância (VI). Os indivíduos de toda comunidade e das seis espécies de maior VI foram distribuídos em classes diamétricas, onde o número de classes (k) e a amplitude (c) foram determinados pelo método estatístico. Esse método utilizou os critérios (FERREIRA, 2005): I) se o número de indivíduos amostrados (n) na comunidade, ou em cada população, foi $\leq 100$, $\mathrm{k}=\sqrt{n} ;$ se $\mathrm{n}>100 ; \mathrm{k}=5 \log (\mathrm{n})$, sendo o valor resultante de $\mathrm{k}$ arredondado para o número inteiro mais próximo; II) a amplitude das classes (c) fixas dentro da comunidade e de cada população foi determinada por:

$$
c=\frac{>o b s-<o b s}{k-1}
$$

Sendo $>$ obs $=$ maior valor observado de diâmetro na comunidade e dentro de cada população; $<$ obs $=$ menor valor observado de diâmetro na comunidade e dentro de cada população.

Para o estudo das relações florísticas, entre o fragmento estudado e os demais remanescentes no Planalto Sul-Catarinense, foram compiladas da literatura científica existente e retiradas de dados ainda não publicados, as listagens florísticas de oito áreas: Morro da Igreja (FALKENBERG, 2003); Serra do Corvo (FALKENBERG, 2003); Serra do Rio do Rastro (FALKENBERG, 2003); Campo Belo do Sul (FORMENTO et al., 2004); Bom Jardim da Serra (ESKUCHE, 2007); Campos Novos (HIGUCHI et al., dados não publicados); Lages, na localidade de Pedras Brancas (HIGUCHI et al., dados não publicados); e Painel (HIGUCHI et al., dados não publicados). Foram extraídas das listagens florísticas apenas espécies arborescentes, as quais passaram por uma revisão das sinonímias botânicas. Os dados de variáveis ambientais (climáticas de 1960-1990 e geográficas, referentes à altitude, à longitude e à latitude) referentes a cada uma dessas áreas foram extraídos do banco de dados do WORLDCLIM (HIJMANS et al., 2005). As variáveis climáticas utilizadas foram: temperatura média anual, média da amplitude térmica diária, isotermalidade, sazonalidade térmica (desvio padrão da temperatura média mensal), temperatura máxima no mês mais quente, temperatura mínima no mês mais frio, amplitude térmica anual, temperatura média no trimestre mais úmido, temperatura no trimestre mais úmido, temperatura média no trimestre mais quente, temperatura média no trimestre mais frio, precipitação total anual, precipitação no mês mais úmido, precipitação no mês mais seco, sazonalidade da precipitação, precipitação no trimestre mais úmido, precipitação no trimestre mais seco, precipitação no trimestre mais quente e precipitação no trimestre mais frio. A relação florística entre as áreas, em função da heterogeneidade ambiental existente, foi analisada por meio da Árvore de Regressão Multivariada (ARM) (DE'ATH, 2002), feita no programa estatístico R (R DEVELOPMENT CORE TEAM, 2009), utilizando a biblioteca MVPART (DE'ATH, 2006). Como variável dependente foi utilizada uma matriz de dissimilaridade, calculada pelo método de Sorensen, e como independentes foram consideradas as variáveis climáticas e geográficas (latitude, longitude e altitude). Uma árvore de regressão é constituída por nós e ramos, sendo o nó inicial formado pela amostra original (FARIA et al., 2010). A partir do nó inicial, a comunidade arbórea é particionada em cada nó intermediário em grupos mais homogêneos, baseando-se na similaridade florística entre as áreas e nas variáveis ambientais associadas. Além disso, as variáveis significativas e seus respectivos valores limites (Threshold values) são fornecidos para cada partição da comunidade, determinando onde cada área ocorre ao longo do 
gradiente ambiental (DE'ATH, 2006). O tamanho da árvore de regressão, ou seja, a quantidade de subdivisões dos nós, foi definido de forma a produzir o menor erro relativo obtido pela validação cruzada (ERVD). O ajuste da ARM foi verificado pelo erro relativo (ER), que indica quanto da variância não foi explicada. Para cada grupo definido pela árvore de regressão, foram verificadas quais eram as espécies exclusivas e com ocorrência em todas as áreas que formavam o grupo (frequência de 100\%).

\section{RESULTADOS}

Foram identificadas 87 espécies arbóreas, pertencentes a 58 gêneros e a 36 famílias botânicas (Tabela 1). A diversidade, representada pelo índice de Shannon, e a dominância ecológica, indicada pelo índice de Pielou, tiveram valores de: $\mathrm{H}^{\prime}=$ 3,6 e J' $=0,82$. As famílias com maior número de espécies foram Myrtaceae (18), Asteraceae (6), Lauraceae (5) e Salicaceae (5). Os gêneros de maior riqueza foram Myrcia (6), Eugenia (4), Ilex
(4), Maytenus (3) e Myrsine (3).

Dentro das parcelas foram amostrados 1.783 indivíduos distribuídos em 80 espécies arbóreas, pertencentes a 54 gêneros e a 35 famílias botânicas, ou seja, somente sete espécies foram encontradas fora das parcelas. As 80 espécies encontradas dentro das parcelas representam $92,21 \%$ a $95,85 \%$ da riqueza esperada pelos estimadores Jackknife de primeira e segunda ordem, que tiveram valores de 86,76 e 83,46 espécies, respectivamente.

A área basal total das espécies em 1 ha foi de $35,54 \mathrm{~m}^{2}$, sendo que as espécies de maior VI foram Araucaria angustifolia e Podocarpus lambertii (Tabela 2). As espécies mais raras, ou seja, aquelas que apresentaram somente um indivíduo amostrado, foram: Lamanonia ternata, Quillaja brasiliensis, Ilex dumosa, Myrcengenia euosma, Daphnopsis racemosa e Maytenus boaria. Também na Tabela 2 é possível verificar que as alturas médias e máximas destas e das outras populações tiveram padrões diferenciados.

TABELA 1: Espécies arbóreas encontradas em um fragmento de Floresta Ombrófila Mista Montana em Lages, SC, com seus respectivos números de registro (R) no Herbário LUSC.

TABLE 1: Tree species found in a montane Araucaria Forest fragment in Lages, SC state with their respective registry number in LUSC Herbarium.

\begin{tabular}{|c|c|c|c|c|c|}
\hline Famílias/Espécies & $\mathrm{L}$ & $\mathrm{R}$ & Famílias/Espécies & $\mathrm{L}$ & $\mathrm{R}$ \\
\hline ANACARDIACEAE & & & MYRTACEAE & & \\
\hline Lithraea brasiliensis Marchand & $\mathrm{P}$ & 2288 & Acca sellowiana (O.Berg) Burret. & $\mathrm{P}$ & 2320 \\
\hline Schinus polygamus (Cav.) Cabrera & $\mathrm{F}$ & 2291 & Blepharocalyx salicifolius (Kunth.) O.Berg & $\mathrm{P}$ & 2321 \\
\hline Schinus terebinthifolius Raddi & $\mathrm{P}$ & 2290 & Calyptranthes concinna DC. & $\mathrm{P}$ & 2322 \\
\hline ANNONACEAE & & & Campomanesia xanthocarpa O.Berg & $\mathrm{P}$ & 2323 \\
\hline Annona rugulosa (Schltdl.) H.Rainer & $\mathrm{P}$ & 2289 & Eugenia pluriflora DC. & $\mathrm{P}$ & 2330 \\
\hline AQUIFOLIACEAE & & & Eugenia pyriformis Cambess & $\mathrm{P}$ & 2325 \\
\hline Ilex brevicuspis Reissek & $\mathrm{P}$ & 2292 & Eugenia sp. & $\mathrm{P}$ & 2324 \\
\hline Ilex dumosa Reissek & $\mathrm{P}$ & ${ }^{*}$ & Eugenia uruguayensis Cambess & $\mathrm{P}$ & 2326 \\
\hline Ilex paraguariensis A. St.-Hil. & $\mathrm{P}$ & 2294 & Myrceugenia euosma (O.Berg) D.Legrand & $\mathrm{P}$ & 2327 \\
\hline Ilex theezans Mart. ex Reissek & $\mathrm{P}$ & 2293 & $\begin{array}{l}\text { Myrceugenia oxysepala (Burret) D.Legrand } \\
\text { \& Kausel }\end{array}$ & $\mathrm{P}$ & 2328 \\
\hline ARALIACEAE & & & Myrcia bombycina (O.Berg) Nied. & $\mathrm{P}$ & 2329 \\
\hline Oreopanax fulvus Marchal & $\mathrm{P}$ & 2295 & Myrcia guianensis (Aublet) DC. & $\mathrm{P}$ & 2331 \\
\hline ARAUCARIACEAE & & & Myrcia hatschbachii D.Legrand & $\mathrm{P}$ & 2332 \\
\hline Araucaria angustifolia (Bertol.) Kuntze & $\mathrm{P}$ & 2296 & Myrcia laruotteana Cambess & $\mathrm{P}$ & 2333 \\
\hline ASTERACEAE & & & Myrcia palustris DC. & $\mathrm{P}$ & 2334 \\
\hline Baccharis semiserrata DC. & $\mathrm{F}$ & * & Myrcia splendens (Sw.) DC. & $\mathrm{P}$ & 2335 \\
\hline Baccharis uncinella DC. & $\mathrm{F}$ & * & Myrciaria delicatula (DC.) O.Berg & $\mathrm{P}$ & 2336 \\
\hline
\end{tabular}


TABELA 1: Continuação ...

TABLE 1: Continued ...

\begin{tabular}{|c|c|c|c|c|c|}
\hline Famílias/Espécies & $\mathrm{L}$ & $\mathrm{R}$ & Famílias/Espécies & $\mathrm{L}$ & $\mathrm{R}$ \\
\hline Dasyphyllum spinescens (Less.) Cabrera & $\mathrm{P}$ & 2297 & Myrrhinium atropurpureum Schott & $\mathrm{P}$ & 2337 \\
\hline Dasyphyllum tomentosum (Spreng.) Cabrera & $\mathrm{P}$ & * & PODOCARPACEAE & & \\
\hline Gochnatia polymorpha (Less.) Cabrera & $\mathrm{P}$ & 2298 & Podocarpus lambertii Klotzsch & $\mathrm{P}$ & 2338 \\
\hline Vernonanthura discolor (Spreng.) H.Rob. & $\mathrm{P}$ & 2299 & PRIMULACEAE & & \\
\hline BERBERIDACEAE & & & Myrsine coriacea (Sw.) Roem. \& Schult. & $\mathrm{P}$ & 2317 \\
\hline Berberis laurina Billb. & $\mathrm{F}$ & 2300 & Myrsine gardneriana A. DC. & $\mathrm{P}$ & 2318 \\
\hline BIGNONIACEAE & & & Myrsine umbellata Mart. & $\mathrm{P}$ & 2319 \\
\hline Handroanthus albus (Cham.) Mattos & $\mathrm{P}$ & * & PROTEACEAE & & \\
\hline Jacaranda puberula Cham. & $\mathrm{P}$ & 2301 & Roupala montana Aubl. & $\mathrm{P}$ & 2339 \\
\hline CANELLACEAE & & & QUILLAJACEAE & & \\
\hline Cinnamodendron dinisii Schwacke & $\mathrm{P}$ & 2302 & $\begin{array}{l}\text { Quillaja brasiliensis (A. St.-Hil. \& Tul.) } \\
\text { Mart. }\end{array}$ & $\mathrm{P}$ & * \\
\hline CANNABACEAE & & & RHAMNACEAE & & \\
\hline Celtis brasiliensis (Gardner) Planch. & $\mathrm{P}$ & 2303 & Scutia buxifolia Reissek & $\mathrm{P}$ & 2340 \\
\hline CELASTRACEAE & & & ROSACEAE & & \\
\hline Maytenus boaria Molina & $\mathrm{P}$ & $*$ & Prunus myrtifolia (L.) Urb. & $\mathrm{P}$ & 2341 \\
\hline Maytenus evonymoides Reissek & $\mathrm{P}$ & 2304 & RUTACEAE & & \\
\hline Maytenus muelleri Schwacke & $\mathrm{P}$ & 2305 & $\begin{array}{l}\text { Zanthoxylum kleinii (R.S.Cowan) } \\
\text { P.G.Waterman }\end{array}$ & $\mathrm{P}$ & * \\
\hline CLETHRACEAE & & & Zanthoxylum rhoifolium Lam. & $\mathrm{P}$ & 2342 \\
\hline Clethra scabra Pers. & $\mathrm{P}$ & 2306 & SALICACEAE & & \\
\hline CUNONIACEAE & & & Banara tomentosa Clos & $\mathrm{P}$ & 2343 \\
\hline Lamanonia ternata Vell. & $\mathrm{P}$ & * & Casearia decandra Jacq. & $\mathrm{P}$ & 2344 \\
\hline DICKSONIACEAE & & & Casearia obliqua Spreng. & $\mathrm{P}$ & * \\
\hline Dicksonia sellowiana Hook. & $\mathrm{P}$ & * & Xylosma ciliatifolia (Clos) Eichler & $\mathrm{P}$ & $*$ \\
\hline ERYTHROXYLACEAE & & & Xylosma tweediana (Clos) Eichler & $\mathrm{P}$ & 2345 \\
\hline $\begin{array}{l}\text { Erythroxylum cuneifolium (Mart.) } \\
\text { O.E.Schulz }\end{array}$ & $\mathrm{P}$ & 2307 & SAPINDACEAE & & \\
\hline Erythroxylum deciduum A. St.-Hil. & $\mathrm{P}$ & 2308 & Allophylus guaraniticus (A. St.-Hil.) Radlk. & $\mathrm{P}$ & 2346 \\
\hline EUPHORBIACEAE & & & Cupania vernalis Cambess & $\mathrm{P}$ & 2347 \\
\hline Sapium glandulosum (L.) Morong & $\mathrm{P}$ & * & Matayba elaeagnoides Radlk. & $\mathrm{P}$ & 2348 \\
\hline Sebastiania brasiliensis Spreng. & $\mathrm{P}$ & 2309 & SOLANACEAE & & \\
\hline $\begin{array}{l}\text { Sebastiania commersoniana (Baill.) L. B. } \\
\text { Sm. \& Downs }\end{array}$ & $\mathrm{P}$ & 2310 & Brunfelsia cuneifolia J.A.Shimidt & $\mathrm{F}$ & 2349 \\
\hline FABACEAE & & & Solanum pabstii L.B.Sm. \& Downs & $\mathrm{P}$ & 2350 \\
\hline Dalbergia frutescens (Vell.) Britton & $\mathrm{P}$ & * & Solanum sanctaecatharinae Dunal & $\mathrm{P}$ & 2351 \\
\hline LAURACEAE & & & STYRACACEAE & & \\
\hline Nectandra lanceolata Nees & $\mathrm{P}$ & 2314 & Styrax acuminatus Pohl & $\mathrm{P}$ & 2352 \\
\hline Nectandra megapotamica (Spreng.) Mez & $\mathrm{P}$ & 2311 & Styrax leprosus Hook. \& Arn. & $\mathrm{P}$ & 2353 \\
\hline Ocotea puberula (Rich.) Nees & $\mathrm{P}$ & * & SYMPLOCACEAE & & \\
\hline Ocotea pulchella Mart. & $\mathrm{P}$ & 2312 & Symplocos uniflora (Pohl) Benth. & $\mathrm{P}$ & 2354 \\
\hline Persea major L.E.Kopp & $\mathrm{P}$ & 2313 & THYMELAEACEAE & & \\
\hline LOGANIACEAE & & & Daphnopsis racemosa Griseb. & $\mathrm{P}$ & 2355 \\
\hline Strychnos brasiliensis (Spreng.) Mart. & $\mathrm{P}$ & 2315 & VERBENACEAE & & \\
\hline
\end{tabular}


TABELA 1: Continuação ...

TABLE 1: Continued ...

\begin{tabular}{|c|c|c|c|c|c|}
\hline Famílias/Espécies & $\mathrm{L}$ & $\mathrm{R}$ & Famílias/Espécies & $\mathrm{L}$ & $\mathrm{R}$ \\
\hline MELASTOMATACEAE & & & Duranta vestita Cham. & $\mathrm{P}$ & 2356 \\
\hline Leandra regnellii (Triana) Cogn. & $\mathrm{F}$ & * & WINTERACEAE & & \\
\hline Miconia cinerascens Miq. & $\mathrm{P}$ & 2316 & Drimys brasiliensis Miers & $\mathrm{P}$ & 2357 \\
\hline $\begin{array}{l}\text { Miconia hiemalis A.St.-Hil. \& Naudin ex } \\
\text { Naudin }\end{array}$ & $\mathrm{F}$ & * & & & \\
\hline
\end{tabular}

Em que: $\mathrm{L}=$ local onde a espécie foi encontrada, sendo $\mathrm{P}$ as espécies amostradas nas parcelas e $\mathrm{F}$ as espécies registradas somente na florística; ${ }^{*}=$ sem coleta.

TABELA 2: Espécies arbóreas encontradas nas 25 parcelas alocadas em um fragmento de Floresta Ombrófila Mista Montana em Lages, SC, ordenadas pelo Valor de Importância (VI).

TABLE 2: Tree species within 25 plots allocated in a montane Araucaria Forest fragment in Lages, SC state, ranked by Importance Value (VI).

\begin{tabular}{|c|c|c|c|c|c|c|c|c|c|}
\hline Espécie & $\mathrm{H}$ & $\mathrm{Hx}$ & DA & DR & DoA & DoR & FA & FR & VI \\
\hline Araucaria angustifolia & 11,0 & 25 & 198 & 11,10 & 4,685 & 13,18 & 100 & 4,19 & 9,49 \\
\hline Podocarpus lambertii & 8,6 & 20 & 139 & 7,80 & 2,388 & 6,72 & 96 & 4,03 & 6,18 \\
\hline Myrcia splendens & 8,0 & 18 & 159 & 8,92 & 0,999 & 2,81 & 88 & 3,69 & 5,14 \\
\hline Matayba elaeagnoides & 12,7 & 20 & 47 & 2,64 & 2,953 & 8,31 & 56 & 2,35 & 4,43 \\
\hline Zanthoxylum kleinii & 10,9 & 20 & 76 & 4,26 & 2,174 & 6,12 & 68 & 2,85 & 4,41 \\
\hline Ocotea pulchella & 13,3 & 20 & 36 & 2,02 & 2,902 & 8,17 & 68 & 2,85 & 4,35 \\
\hline Jacaranda puberula & 8,5 & 17 & 103 & 5,78 & 1,179 & 3,32 & 60 & 2,52 & 3,87 \\
\hline Lithraea brasiliensis & 10,6 & 16 & 35 & 1,96 & 1,963 & 5,52 & 60 & 2,52 & 3,33 \\
\hline Casearia decandra & 6,6 & 12 & 74 & 4,15 & 0,546 & 1,54 & 92 & 3,86 & 3,18 \\
\hline Campomanesia xanthocarpa & 9,1 & 16 & 55 & 3,08 & 0,970 & 2,73 & 68 & 2,85 & 2,89 \\
\hline Myrsine umbellata & 7,9 & 14 & 58 & 3,25 & 0,581 & 1,64 & 84 & 3,52 & 2,80 \\
\hline Dicksonia sellowiana & 2,5 & 5 & 39 & 2,19 & 1,669 & 4,69 & 20 & 0,84 & 2,57 \\
\hline Scutia buxifolia & 7,0 & 15 & 61 & 3,42 & 0,598 & 1,68 & 60 & 2,52 & 2,54 \\
\hline Myrcia bombycina & 6,4 & 15 & 78 & 4,37 & 0,649 & 1,83 & 32 & 1,34 & 2,51 \\
\hline Banara tomentosa & 6,9 & 12 & 51 & 2,86 & 0,363 & 1,02 & 64 & 2,68 & 2,19 \\
\hline Myrcia guianensis & 7,1 & 15 & 33 & 1,85 & 0,388 & 1,09 & 80 & 3,36 & 2,10 \\
\hline Nectandra megapotamica & 12,8 & 18 & 16 & 0,90 & 1,345 & 3,78 & 36 & 1,51 & 2,06 \\
\hline Allophylus guaraniticus & 7,1 & 10 & 29 & 1,63 & 0,502 & 1,41 & 60 & 2,52 & 1,85 \\
\hline Duranta vestita & 5,5 & 8,5 & 40 & 2,24 & 0,304 & 0,86 & 56 & 2,35 & 1,82 \\
\hline Vernonanthura discolor & 10,7 & 18 & 22 & 1,23 & 0,620 & 1,74 & 48 & 2,01 & 1,66 \\
\hline Dasyphyllum spinescens & 10,5 & 17 & 24 & 1,35 & 0,677 & 1,90 & 32 & 1,34 & 1,53 \\
\hline Symplocos uniflora & 9,1 & 15 & 20 & 1,12 & 0,380 & 1,07 & 44 & 1,85 & 1,35 \\
\hline Roupala Montana & 10,1 & 18 & 15 & 0,84 & 0,536 & 1,51 & 40 & 1,68 & 1,34 \\
\hline Cupania vernalis & 10,9 & 20 & 14 & 0,79 & 0,548 & 1,54 & 24 & 1,01 & 1,11 \\
\hline Myrrhinium atropurpureum & 6,9 & 12 & 17 & 0,95 & 0,134 & 0,38 & 40 & 1,68 & 1,00 \\
\hline Dasyphyllum tomentosum & 11,5 & 18 & 11 & 0,62 & 0,402 & 1,13 & 28 & 1,17 & 0,97 \\
\hline Solanum sanctaecatharinael & 8,6 & 16 & 13 & 0,73 & 0,157 & 0,44 & 36 & 1,51 & 0,89 \\
\hline Sebastiania commersoniana & 10,3 & 18 & 13 & 0,73 & 0,447 & 1,26 & 16 & 0,67 & 0,89 \\
\hline Annona rugulosa & 6,4 & 12 & 15 & 0,84 & 0,105 & 0,30 & 36 & 1,51 & 0,88 \\
\hline Drimys brasiliensis & 6,3 & 9 & 13 & 0,73 & 0,087 & 0,24 & 32 & 1,34 & 0,77 \\
\hline
\end{tabular}


TABELA 2: Continuação ...

TABLE 2: Continued ...

\begin{tabular}{|c|c|c|c|c|c|c|c|c|c|}
\hline Espécie & $\mathrm{H}$ & $\mathrm{Hx}$ & DA & DR & DoA & DoR & FA & FR & VI \\
\hline Zanthoxylum rhoifolium & 9,0 & 16 & 12 & 0,67 & 0,166 & 0,47 & 28 & 1,17 & 0,77 \\
\hline Gochnatia polymorpha & 8,4 & 12 & 8 & 0,45 & 0,275 & 0,77 & 24 & 1,01 & 0,74 \\
\hline Cinnamodendron dinisii & 6,9 & 12 & 13 & 0,73 & 0,115 & 0,32 & 28 & 1,17 & 0,74 \\
\hline Eugenia pyriformis & 11,8 & 16 & 9 & 0,50 & 0,123 & 0,35 & 32 & 1,34 & 0,73 \\
\hline Myrcia palustris & 6,0 & 8 & 11 & 0,62 & 0,110 & 0,31 & 28 & 1,17 & 0,70 \\
\hline Oreopanax fulvus & 9,1 & 15 & 7 & 0,39 & 0,143 & 0,40 & 28 & 1,17 & 0,66 \\
\hline Xylosma ciliatifolia & 6,9 & 10 & 10 & 0,56 & 0,078 & 0,22 & 28 & 1,17 & 0,65 \\
\hline Calyptranthes concinna & 5,5 & 7 & 9 & 0,50 & 0,077 & 0,22 & 28 & 1,17 & 0,63 \\
\hline Nectandra lanceolata & 11,8 & 14 & 4 & 0,22 & 0,404 & 1,14 & 12 & 0,50 & 0,62 \\
\hline Sebastiania brasiliensis & 4,3 & 6 & 17 & 0,95 & 0,179 & 0,50 & 8 & 0,34 & 0,60 \\
\hline Casearia obliqua & 7,9 & 11 & 10 & 0,56 & 0,135 & 0,38 & 20 & 0,84 & 0,59 \\
\hline Styrax leprosus & 8,4 & 17 & 10 & 0,56 & 0,130 & 0,37 & 20 & 0,84 & 0,59 \\
\hline Dalbergia frutescens & 9,2 & 13 & 9 & 0,50 & 0,084 & 0,24 & 24 & 1,01 & 0,58 \\
\hline Erythroxylum cuneifolium & 7,5 & 12 & 11 & 0,62 & 0,119 & 0,33 & 16 & 0,67 & 0,54 \\
\hline Solanum pabstii & 6,9 & 12 & 8 & 0,45 & 0,035 & 0,10 & 24 & 1,01 & 0,52 \\
\hline Acca sellowiana & 5,6 & 9 & 8 & 0,45 & 0,085 & 0,24 & 20 & 0,84 & 0,51 \\
\hline Ocotea puberula & 13,3 & 21 & 5 & 0,28 & 0,199 & 0,56 & 16 & 0,67 & 0,50 \\
\hline Ilex paraguariensis & 11,8 & 20 & 4 & 0,22 & 0,239 & 0,67 & 12 & 0,50 & 0,47 \\
\hline Myrsine coriacea & 11,2 & 15 & 5 & 0,28 & 0,097 & 0,27 & 20 & 0,84 & 0,46 \\
\hline Prunus myrtifolia & 9,1 & 18 & 7 & 0,39 & 0,109 & 0,31 & 16 & 0,67 & 0,46 \\
\hline Ilex theezans & 8,4 & 13 & 6 & 0,34 & 0,067 & 0,19 & 20 & 0,84 & 0,46 \\
\hline Eugenia pluriflora & 6,9 & 9,5 & 6 & 0,34 & 0,047 & 0,13 & 20 & 0,84 & 0,44 \\
\hline Blepharocalyx salicifolius & 7,8 & 12 & 6 & 0,34 & 0,033 & 0,09 & 20 & 0,84 & 0,42 \\
\hline Myrcia hatschbachii & 7,3 & 10 & 5 & 0,28 & 0,044 & 0,12 & 20 & 0,84 & 0,41 \\
\hline Maytenus muelleri & 8,0 & 10 & 5 & 0,28 & 0,100 & 0,28 & 16 & 0,67 & 0,41 \\
\hline Ilex brevicuspis & 11,0 & 18 & 4 & 0,22 & 0,097 & 0,27 & 16 & 0,67 & 0,39 \\
\hline Eugenia sp. & 6,1 & 9 & 6 & 0,34 & 0,052 & 0,15 & 16 & 0,67 & 0,39 \\
\hline Handroanthus albus & 8,5 & 13 & 4 & 0,22 & 0,178 & 0,50 & 8 & 0,34 & 0,35 \\
\hline Celtis brasiliensis & 5,7 & 12 & 6 & 0,34 & 0,067 & 0,19 & 12 & 0,50 & 0,34 \\
\hline Myrcia laruotteana & 7,0 & 13 & 5 & 0,28 & 0,024 & 0,07 & 16 & 0,67 & 0,34 \\
\hline Strychnos brasiliensis & 2,7 & 4 & 4 & 0,22 & 0,013 & 0,04 & 16 & 0,67 & 0,31 \\
\hline Maytenus evonymoides & 11,7 & 18 & 4 & 0,22 & 0,110 & 0,31 & 8 & 0,34 & 0,29 \\
\hline Clethra scabra & 6,8 & 10 & 4 & 0,22 & 0,048 & 0,14 & 12 & 0,50 & 0,29 \\
\hline Erythroxylum deciduum & 8,3 & 10 & 4 & 0,22 & 0,031 & 0,09 & 12 & 0,50 & 0,27 \\
\hline Styrax acuminatus & 5,8 & 8 & 4 & 0,22 & 0,011 & 0,03 & 12 & 0,50 & 0,25 \\
\hline Xylosma tweediana & 5,8 & 7 & 3 & 0,17 & 0,009 & 0,02 & 12 & 0,50 & 0,23 \\
\hline Miconia cinerascens & 5,0 & 6 & 3 & 0,17 & 0,007 & 0,02 & 12 & 0,50 & 0,23 \\
\hline Sapium glandulosum & 13,0 & 20 & 2 & 0,11 & 0,062 & 0,17 & 8 & 0,34 & 0,21 \\
\hline Lamanonia ternata & 12,0 & 12 & 1 & 0,06 & 0,141 & 0,40 & 4 & 0,17 & 0,21 \\
\hline Myrsine gardneriana & 10,0 & 13 & 2 & 0,11 & 0,056 & 0,16 & 8 & 0,34 & 0,20 \\
\hline Eugenia uruguayensis & 6,5 & 7 & 3 & 0,17 & 0,022 & 0,06 & 8 & 0,34 & 0,19 \\
\hline Myrceugenia oxysepala & 6,3 & 7 & 3 & 0,17 & 0,016 & 0,04 & 8 & 0,34 & 0,18 \\
\hline Schinus terebinthifolius & 5,2 & 6 & 3 & 0,17 & 0,013 & 0,04 & 8 & 0,34 & 0,18 \\
\hline
\end{tabular}


TABELA 2: Continuação ...

TABLE 2: Continued ...

\begin{tabular}{lccccccccc}
\hline Espécie & $\mathrm{H}$ & $\mathrm{Hx}$ & $\mathrm{DA}$ & $\mathrm{DR}$ & $\mathrm{DoA}$ & $\mathrm{DoR}$ & $\mathrm{FA}$ & FR & VI \\
\hline Persea major & 10,5 & 13 & 2 & 0,11 & 0,025 & 0,07 & 8 & 0,34 & 0,17 \\
Myrciaria delicatula & 8,0 & 10 & 2 & 0,11 & 0,019 & 0,05 & 8 & 0,34 & 0,17 \\
Quillaja brasiliensis & 10,0 & 10 & 1 & 0,06 & 0,066 & 0,19 & 4 & 0,17 & 0,14 \\
Ilex dumosa & 10,0 & 10 & 1 & 0,06 & 0,021 & 0,06 & 4 & 0,17 & 0,09 \\
Myrceugenia euosma & 6,5 & 6,5 & 1 & 0,06 & 0,006 & 0,02 & 4 & 0,17 & 0,08 \\
Daphnopsis racemosa & 5,0 & 5 & 1 & 0,06 & 0,004 & 0,01 & 4 & 0,17 & 0,08 \\
Maytenus boaria & 5,0 & 5 & 1 & 0,06 & 0,002 & 0,01 & 4 & 0,17 & 0,08 \\
\hline Total & & & 1783 & 100,0 & 35,54 & 100,00 & 2384 & 100,0 & 100,0 \\
\hline
\end{tabular}

Em que: $\mathrm{H}=$ altura média de cada espécie $(\mathrm{m}) ; \mathrm{Hx}=$ altura máxima de cada espécie $(\mathrm{m})$; DA = densidade absoluta (ind ha-1 $) ; \mathrm{DR}=$ densidade relativa $(\%) ; \mathrm{DoA}=$ dominância absoluta $\left(\mathrm{m}^{2} \mathrm{ha}^{-1}\right)$; DoR $=$ dominância relativa $(\%) ; \mathrm{FA}=$ frequência absoluta $(\%) ; \mathrm{FR}=$ frequência relativa $(\%)$; VI = valor de importância $(\%)$.

A distribuição diamétrica da comunidade teve um padrão próximo ao $\mathrm{J}$ invertido (Figura 2), enquanto que a distribuição diamétrica, das populações das seis espécies de maior VI, apresentouse de diferentes formas (Figura 3): Araucaria angustifolia, Podocarpus lambertii e Zanthoxylum kleinii demonstraram padrão próximo ao J invertido, e Myrcia splendens, Matayba elaeagnoides e Ocotea pulchella tiveram padrões diferentes do J invertido, com distribuição descontínua nas classes diamétricas ou com muitos indivíduos em maiores classes de diâmetro.

A Árvore de Regressão Multivariada (ARM) (Figura 4), com um ERVD de 0,621, explicou 83,3\% da variação encontrada $(E R=0,167)$ e demonstra que o fragmento estudado ocorreu no agrupamento florístico junto com Campo Belo do Sul, Campos Novos e Lages (localidade de Pedras Brancas). A variável ambiental, que separou este grupo dos

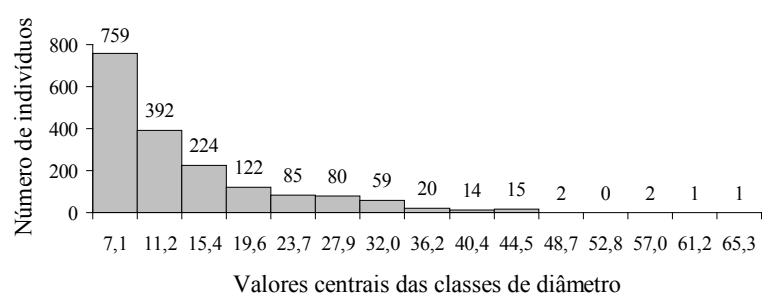

FIGURA 2: Estrutura diamétrica da comunidade nas 25 parcelas alocadas em um fragmento de Floresta Ombrófila Mista Montana em Lages, SC.

FIGURE 2: Tree community diametric structure within 25 plots allocated in a montane Araucaria Forest fragment in Lages, SC. demais, foi a sazonalidade de temperatura maior ou igual a $3,053^{\circ} \mathrm{C}$. As espécies exclusivas do grupo e com ocorrência em todas as áreas deste foram: Casearia decandra, Cinnamodendron dinisii, Cupania vernalis, Sebastiania commersoniana, Ilex dumosa, Jacaranda puberula, Lithraea brasiliensis, Matayba elaignoides, Myrsine umbellata e Sebastiania brasiliensis. Nos locais onde a sazonalidade de temperatura foi menor do que $3,053^{\circ} \mathrm{C}$, foram formados os demais agrupamentos. $\mathrm{O}$ segundo grupo (áreas: Morro da Igreja, Serra do Corvo e Serra do Rio do Rastro) teve a temperatura média anual menor que $13,8^{\circ} \mathrm{C}$. As espécies exclusivas desse grupo e com ocorrência nas três áreas foram: Agarista niederleinii (Sleumer) Judd, Austroeupatorium inulifolium (Kunth) R.M.King \& H.Rob., Azara uruguayensis (Speg.) Sleumer, Baccharis oblongifolia (Ruiz \& Pav.) Pers., Baccharis retusa DC., Escallonia petrophila Rambo \& Sleumer, Eugenia handroi (Mattos) Mattos, Gaylussacia brasiliensis (Spreng.) Mart., Leandra quinquedentata (DC.) Cogn., Miconia ramboi Brade, Mimosa taimbensis Burkart, Myrcengenia alpigena (DC.) Landrum, Myrcengenia pilotantha (Kiaersk.) Landrum, Myrciaria floribunda (H.West ex Willd.) O.Berg, Roupala asplenioides Sleumer, Siphoneugena reitzii D.Legrand e Symplocos itatiaiae Wawra. O terceiro grupo, com temperatura média anual maior ou igual a $13,8^{\circ} \mathrm{C}$, teve como localidades representantes Bom Jardim da Serra e Painel, com somente uma espécie exclusiva e com ocorrência em ambas as áreas: Symphyopappus itatiayensis (Hieron.) R.M.King \& H.Rob. As espécies que ocorreram em todas as áreas dos três grupos foram somente Araucaria angustifolia, Drimys brasiliensis, Ocotea pulchela e Prunus myrtifolia. 

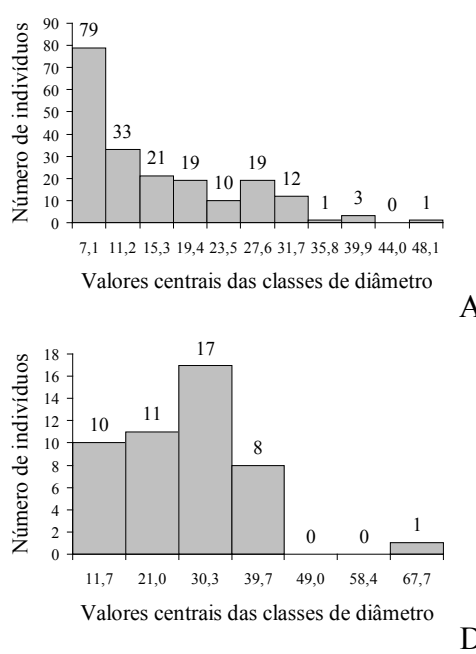
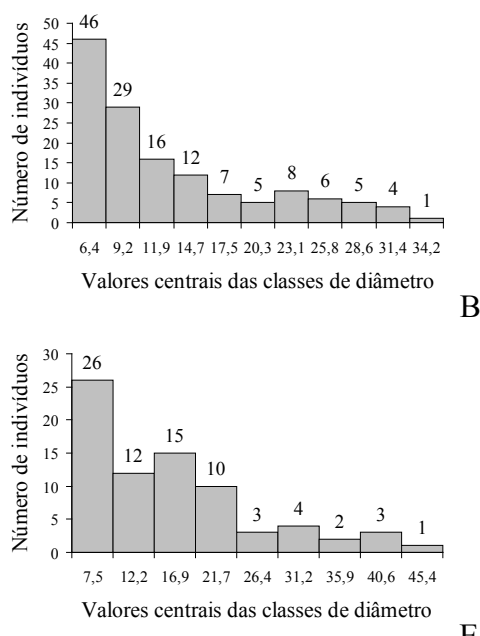
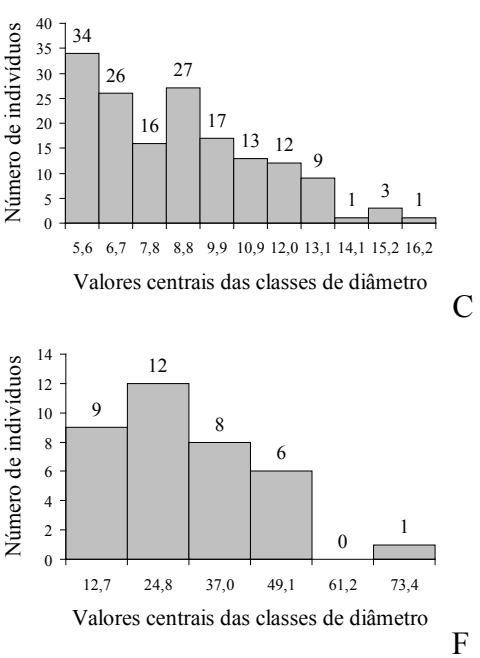

FIGURA 3: Estrutura diamétrica das seis espécies de maior Valor de Importância (VI) - Araucaria angustifolia (A), Podocarpus lambertii (B), Myrcia splendens (C), Matayba elaeagnoides (D), Zanthoxylum kleinii (E) e Ocotea pulchella (F) - nas 25 parcelas alocadas em um fragmento de Floresta Ombrófila Mista Montana em Lages, SC.

FIGURE 3: Diametric structure of the species with highest Importannce Value (VI) - Araucaria angustifolia (A), Podocarpus lambertii (B), Myrcia splendens (C), Matayba elaeagnoides (D), Zanthoxylum kleinii (E) e Ocotea pulchella $(\mathrm{F})$ - within 25 plots allocated in a montane Araucaria Forest fragment in Lages, SC.

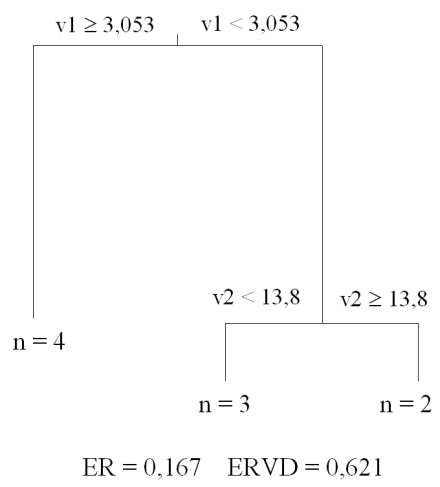

FIGURA 4: Árvore de Regressão Multivariada realizada para a matriz de similaridade entre os remanescentes florestais localizados no Planalto Sul-Catarinense, em função da heterogeneidade ambiental existente (geográfica e climática). No final de cada ramificação está indicado o número de áreas $(n)$ que se enquadra em cada condição ambiental, sendo o primeiro grupo $(n=4)$ formado por Campo Belo do Sul, Campos Novos, Lages - localidade Pedras Brancas e Lages - presente estudo; o segundo grupo $(\mathrm{n}=3)$ formado por Morro da Igreja, Serra do Corvo e Serra do Rio do Rastro; e o terceiro grupo $(\mathrm{n}=2)$ formado por Bom Jardim da Serra e Painel. v1 = sazonalidade térmica $\left({ }^{\circ} \mathrm{C}\right) ; \mathrm{v} 2=$ temperatura média anual $\left({ }^{\circ} \mathrm{C}\right) ; \mathrm{ER}=$ erro relativo e ERVD $=$ erro relativo produzido pela validação cruzada.

FIGURE 4: Multivariate Regression Tree produced to the similarity matrix among forest remnants located in Santa Catarina Southern Plateau, in function of environmental heterogeneity (geographic and climate). At the end of each branch is indicated the number of areas (n) that fall under each environmental condition. The first group $(\mathrm{n}=4)$ formed by Campo Belo do Sul, Campos Novos, Lages - Pedras Brancas and Lages - this study. The second group $(n=3)$ is formed by Morro da Igreja, Serra do Corvo and Serra do Rio do Rastro. The Third group $(n=2)$ is formed by Bom Jardim da Serra and Painel. v1 = Temperature Seasonality $\left({ }^{\circ} \mathrm{C}\right)$; v2 = Annual Mean Temperature $\left({ }^{\circ} \mathrm{C}\right) ; \mathrm{ER}=$ relative error and $\mathrm{ERVD}=$ cross-validated relative error. 


\section{DISCUSSÃO}

O número total de espécies encontrado (87) e a diversidade $\left(\mathrm{H}^{\prime}=3,6\right)$ foram valores altos quando comparados com outros estudos em Floresta Ombrófila Mista que também sofreu impacto antrópico, como o de Nascimento et al. (2001), que encontrou 54 espécies e $\mathrm{H}^{\prime}=3,0$ (nível de inclusão de DAP $\geq 9,6 \mathrm{~cm}$ ). Além disso, no presente estudo, a equabilidade de Pielou foi relativamente elevada $\left(\mathrm{J}^{\prime}=0,82\right)$, o que indica baixa dominância ecológica e uma distribuição uniforme de indivíduos entre as espécies, ao contrário do que seria esperado para florestas em início de sucessão, em que a dominância é alta (CONDIT, 2004). Destaca-se também a ausência da ocorrência de Mimosa scabrella Benth., considerada uma espécie típica da Floresta Ombrófila Mista em início de estádio sucessional pós-distúrbio. Assim, a elevada riqueza de espécies, associada à baixa dominância ecológica e à ausência de algumas espécies tipicamente pioneiras, sugere que a floresta apresenta-se em estágio de sucessão avançado, associado às características intrínsecas da área, como a elevada biodiversidade residual apesar do corte seletivo - e a grande heterogeneidade ambiental observada no campo, com áreas de encosta e de margens de curso de água, bem como o histórico do impacto.

A amostragem realizada foi adequada, pois o número de espécies encontrado nas unidades amostrais representou $92,21 \%$ e $95,85 \%$ da riqueza esperada pelos estimadores de riqueza Jackknife de primeira e segunda ordem, respectivamente, representando quase a totalidade dos valores dos estimadores.

Myrtaceae, a família com maior número de espécies na floresta estudada (18), é também uma das maiores famílias da flora brasileira (SOUZA e LORENZI, 2005). Além disso, geralmente é a família de maior riqueza de espécies arbóreas na Floresta Ombrófila Mista, conforme demonstrado em outros estudos, como os de Jarenkow e Batista (1987) e Nascimento et al. (2001). Apesar da elevada riqueza da família Myrtaceae no presente estudo, apenas duas espécies desta família estiveram entra as 10 espécies de maior VI, o que representa um padrão comum em florestas tropicais e subtropicais, onde existem poucas espécies com maior densidade e a maioria com poucos indivíduos. Vários fatores, como aqueles dependente da densidade, atuam simultaneamente de forma a controlar o tamanho populacional das árvores nestes tipos florestais. Isto, em parte, explica a elevada diversidade encontrada nestes ecossistemas, uma vez que a menor densidade de uma espécie, necessariamente implica numa menor dominância ecológica no ambiente.

Arancaria angustifolia foi a espécie de maior VI devido ao seu alto número de indivíduos (198), à alta área basal ocupada na área $\left(4,685 \mathrm{~m}^{2}\right)$ e à frequência de $100 \%$ nas unidades amostrais, sendo a única espécie com indivíduos em todas as parcelas. Considerando a elevada heterogeneidade ambiental da área estudada, relacionada com as condições de borda/interior da floresta, topografia e a proximidade com o curso de água, pode-se supor que a espécie apresentada não seja especialista por um determinado tipo de habitat. Além disso, Marchiori (2005) ressalta que, embora exista grande diversidade de angiospermas, as Florestas Ombrófilas Mistas se caracterizam pelo elevado número de indivíduos de Araucaria angustifolia por unidade de área.

Podocarpus lambertii Klotzsch, outra gimnosperma dentre as poucas nativas do Brasil, também está entre as espécies de maior VI (segundo maior VI), com alto número de indivíduos (139), alta frequência nas unidades amostrais (96\%) e área basal também relativamente alta $(2,388$ $\mathrm{m}^{2}$ ). Apesar disso, a área basal de Podocarpus lambertii representa metade da área basal Araucaria angustifolia, por serem indivíduos de menor porte que esta.

Das espécies com maior VI, ainda destacamse as angiospermas: I) Myrcia splendens (terceiro maior VI), que se destacou, principalmente, por seu grande número de indivíduos (159), maior que de Podocarpus lambertii, distribuídos em quase todas as unidades amostrais (88\%), mas que, devido ao menor porte, apresentou baixa área basal $\left(0,999 \mathrm{~m}^{2}\right)$; II) Matayba elaeagnoides (quarto maior VI) que, apesar de poucos indivíduos (47) distribuídos apenas na metade das parcelas (56\%), são representados por árvores de grande porte (área basal $=2,953 \mathrm{~m}^{2}$ ); III) Zanthoxylum kleinii (quinto maior VI), que possui 76 indivíduos relativamente bem distribuídos (68\% das parcelas) e com área basal de 2,174 m²; e IV) Ocotea pulchella (sexto maior VI), com somente 36 indivíduos distribuídos em $68 \%$ das parcelas, porém, com árvores de grande porte (área basal $=2,902 \mathrm{~m}^{2}$ ).

Jarenkow e Batista (1987) constataram a existência de três estratos arbóreos descontínuos na Floresta Ombrófila Mista estudada por eles, sendo o primeiro, com alturas de até $28 \mathrm{~m}$, dominado 
pela Araucaria angustifolia, o segundo, entre 12 e $19 \mathrm{~m}$ de altura, e o terceiro, com altura entre 5 m e $12 \mathrm{~m}$. Neste trabalho, pode ser definido um padrão semelhante de estratificação vertical, com a Araucaria angustifolia dominando o estrato superior, com altura máxima de $25 \mathrm{~m}$, com espécies como Jacaranda puberula ocupando o estrato intermediário, e espécies como Casearia decandra ocupando o estrato inferior. Segundo Hunter Jr. (1990), uma estrutura vertical bem diferenciada, com indivíduos de diferentes tamanhos, oferece elevada diferenciação de nichos, suportando maior biodiversidade.

Ao contrário do que se supunha devido às práticas de roçadas no sub-bosque e pisoteio do gado, a distribuição diamétrica de toda a comunidade, considerando os indivíduos de $5 \mathrm{~cm}$ ou mais de DAP, apresentou o padrão próximo do "J invertido", que é esperado para florestas naturais mais conservadas. Isso pode estar ocorrendo porque as roçadas e o pisoteio do gado não ocorrem de forma homogênea na área, concentrandose predominantemente na borda do fragmento, sendo, no entanto, as parcelas distribuídas em todo o fragmento, possivelmente diluindo o efeito negativo destas práticas.

As populações de Araucaria angustifolia, Podocarpus lambertii e Zanthoxylum kleinii também obtiveram padrões de distribuição diamétrica próximo ao "J invertido", indicando a existência de um estoque regenerativo potencial de árvores a partir de $5 \mathrm{~cm}$ de DAP. Neste tipo de distribuição, a maior parte dos indivíduos ocorre nas classes inferiores de diâmetro, e poucos pertencem às classes superiores (SOLBRIG, 1981), sugerindo, numa situação hipotética de ausência de eventos exógenos de natureza estocástica, a capacidade de perpetuação da população ao longo do tempo. A distribuição decrescente dos diâmetros em estudos de populações de araucária também foi encontrada por Durigan (1999) e Schaaf et al. (2006). Já as populações das espécies Myrcia splendens, Matayba elaeagnoides e Ocotea pulchella demonstraram padrões diferentes do $\mathrm{J}$ invertido, o que poderia indicar ausência de estoque regenerativo ou insuficiência deste, a partir do DAP de $5 \mathrm{~cm}$. É importante ressaltar que uma determinada espécie pode apresentar estoque regenerativo em outras fases de vida, como no banco de plântulas ou sementes. Além disso, espécies que se estabeleceram em outros remanescentes florestais próximos, que possuem fluxo de propágulos contínuo com a área estudada, podem caracterizar metapopulações, o que garantiria a manutenção local de espécies em longo prazo a partir da chegada de propágulos. Schaaf et al. (2006) também destacaram que a pequena quantidade de indivíduos nas classes inferiores de diâmetro nem sempre é um indicativo de degradação populacional. Esses autores, em estudo temporal de uma população de Araucaria angustifolia, obtiveram, em 1979, padrão decrescente, porém, com pequena quantidade de indivíduos nas menores classes diamétricas, sugerindo que a espécie poderia não se manter. Porém, em 2000, a mesma população, além de se manter, aumentou o número de indivíduos. Segundo os autores, uma espécie com poucos indivíduos nas classes inferiores de diâmetro, mas com pequena probabilidade de morrer devido à competição, provavelmente se manterá na floresta, como foi o caso da Araucaria angustifolia, enquanto que, uma espécie com grande parte dos indivíduos sujeitos a morrer devido à competição, necessita apresentar uma grande frequência nas classes diamétricas inferiores para ter chance de sobreviver na comunidade.

A Árvore de Regressão Multivariada demonstrou que a temperatura, representada pela sazonalidade entre os meses e a temperatura média anual, foi a variável mais determinante na definição dos agrupamentos florísticos entre as áreas avaliadas no Planalto Sul-Catarinense. Entre a área mais elevada, no Morro da Igreja $(1.501 \mathrm{~m})$, e a mais baixa, em Campos Novos (711 m), ocorre uma mudança altitudinal de $790 \mathrm{~m}$, o que poderia explicar a variação da temperatura entre áreas localizadas geograficamente próximas entre si. A altitude média do grupo 1, onde está localizada a área de estudo (916 m), é de $894 \mathrm{~m}$, ou seja, menor do que a do Grupo $2(1.440,33 \mathrm{~m})$ e a do Grupo 3 $(1.257,5 \mathrm{~m})$. A maior sazonalidade da temperatura nos locais de menor altitude pode estar associada às maiores temperaturas durante o verão, enquanto que nas áreas de maior altitude a menor sazonalidade poderia estar associada com as temperaturas mais amenas no verão. De fato, no Grupo 1 a temperatura média máxima do mês mais quente foi de $26,5^{\circ} \mathrm{C}$, enquanto nos Grupos 2 e 3 foram de, respectivamente, $22,23^{\circ} \mathrm{C}$ e $23,9^{\circ} \mathrm{C}$. Por outro lado, as temperaturas médias mínimas no mês mais frio nos grupos 1,2 e 3 foram, respectivamente, de $6,6^{\circ} \mathrm{C}$; $5,2^{\circ} \mathrm{C}$ e $5,9^{\circ} \mathrm{C}$. Como já destacado por Box (1995), a sazonalidade de temperatura tem o potencial de controlar a distribuição de tipos vegetacionais, 
pois afeta vários mecanismos fisiológicos, principalmente no que se refere às temperaturas máxima, mínima e na estação de crescimento. Isso pode ser observado no presente estudo em função da elevada substituição de espécie, principalmente entre o Grupo 1 e os Grupos 2 e 3. As espécies Casearia decandra, Cinnamodendron dinisii, Cupania vernalis, Sebastiania commersoniana, Ilex dumosa, Jacaranda puberula, Lithraea brasiliensis, Matayba elaignoides, Myrsine umbellata e Sebastiania brasiliensis, típicas do Grupo 1, são substituídas por Agarista niederleinii, Austroeupatorium inulifolium, Azara uruguayensis, Baccharis oblongifolia, Baccharis retusa, Escallonia petrophila, Eugenia handroi, Gaylussacia brasiliensis, Leandra quinquedentata, Miconia ramboi, Mimosa taimbensis, Myrceugenia alpigena, Myrceugenia pilotantha, Myrciaria floribunda, Roupala asplenioides, Siphoneugena reitzii, Symplocos itatiaiae e Symphyopappus itatiayensis nos Grupos 2 e 3. Por outro lado, foi possível identificar as espécies Araucaria angustifolia, Drimys brasiliensis, Ocotea pulchela e Prunus myrtifolia como supertramps, ou seja, com ampla distribuição geográfica no Planalto SulCatarinense.

\section{CONCLUSÕES}

O conhecimento das relações florísticas e do comportamento fitossociológico do componente arbóreo de florestas secundárias, localizadas em propriedades rurais, representa uma etapa inicial, porém fundamental, para o uso sustentável dos recursos florestais e, consequentemente, para sua conservação.

O levantamento fitossociológico demonstrou que o fragmento estudado apresenta uma elevada riqueza de espécies e está em avançado estágio de sucessão. A espécie com maior valor de importância relativa, Araucaria angustifolia, apresentou um VI menor do que 10\%, o que indica ausência de dominância ecológica. A estrutura diamétrica demonstrou padrão J invertido na comunidade e padrões diferenciados entre as populações. São necessários estudos futuros de dinâmica para verificar possíveis mudanças estruturais na floresta, esperadas em uma floresta secundária.

A área estudada fez parte de um agrupamento florístico que apresentou uma maior sazonalidade da temperatura, associadas às áreas de menor altitude no Planalto Sul-Catarinense.

\section{REFERÊNCIAS BIBLIOGRÁFICAS}

APG III. An update of the Angiosperm Phylogeny Group classification for the orders and families of flowering plants: APG III. Botanical Journal of the Linnean Society, London, v. 161, n. 2, p. 105121, 2009.

BOX, E. O. Factors determining distribution of tree species and plant functional types. Plant Ecology, Dordrecht, v. 121, p. 101-116, 1995.

BRASIL, Ministério da agricultura e reforma agrária. Normais climatológicas 1961-1990. Brasília: MARA, 1992. 84 p.

BROWER, J. E.; ZAR, J. H. Field and laboratory methods for general ecology. Duduque: W.M.C. Brow Publishers. 1984. 84 p.

BROWN, S.; LUGO, A. E. Tropical secondary forests. Journal of Tropical Ecology, Cambridge, v. 6, n. 1, p. 1-32, 1990.

CONDIT, R. Local variation in canopy disturbance and soil structure. In: LOSOS, E. C.; LEIGH-JR, E. G. Tropical forest diversity and dynamism: findings from a large-scale plot network. Chicago: The University of Chicago Press, 2004. p. 175-186. CORLETT, R. T. What is secondary Forest? Journal of Tropical Ecology, Cambridge, v. 10, n. 3, p. 445-447, Aug. 1994.

DE'ATH, G. Multivariate regressions trees: a new technique for modeling species-environment relationships. Ecology, Ithaca, v. 83, n. 4, p. 11051117, 2002.

DE'ATH, G. mvpart: Multivariate partitioning. R package version 1.2-4 2006.

DURIGAN, M. E. Florística, dinâmica e análise protéica de uma Floresta Ombrófila Mista em São João do Triunfo - PR. 1999. 125 f. Dissertação (Mestrado em Engenharia Florestal) - Universidade Federal do Paraná, Curitiba, 1999.

ESKUCHE, U. El bosque de Araucaria con Podocarpus y los campos de Bom Jardim da Serra, Santa Catarina (Brasil meridional). Boletín de la Sociedad Argentina de Botánica, Córdoba, v. 42, n. 3-4, p. 295-308, ago./dez. 2007.

FALKENBERG, D. Matinhas nebulares e vegetação rupícola dos Aparados da Serra Geral (SC/RS), sul do Brasil. 2003. 558 f. Tese (Doutorado em Biologia Vegetal) - Universidade Estadual de Campinas, Campinas, 2003.

FARIA, R. N. et al. Avaliação das exigências técnicas às exportações brasileiras de alimentos 
por árvores de classificação multivariadas. Estudos Econômicos, São Paulo, v. 40, n. 3, set. 2010. FERREIRA, D. F. Estatística Básica. Lavras: Ed. da UFLA. 2005. 664 p.

FINEGAN, B. The management potential of neotrocipal lowland rain forest. Forest Ecology and Management, Amsterdam, v. 47, p. 295-321, 1992.

FORMENTO, S. et al. Dinâmica estrutural arbórea de uma Floresta Ombrófila Mista em Campo Belo do Sul, SC. Cerne, Lavras, v. 10, n. 2, p. 196-212, jul./dez. 2004.

GREIG-SMITH, P. Ecological observation on degraded and secondary forest in Trinidad, British West Indies. General feature of the vegetation. Journal of Ecology, Oxford, v. 40, p. 283-315, 1952.

HELTSHE, J. F.; FORRESTER, N. E. Estimating species richness using the jackknife procedure. Biometrics, New York, v. 39, p. 1-12, 1983.

HIJMANS, R. et al. Very high resolution interpolated climate surfaces for global land areas. International Journal of Climatology, Malden, v. 25, n. 15, p. 1965-1978, 2005.

HUNTER Jr, M. L. Wildlife forests and forestry: principles of managing forests for biological diversity. New Jersey: Prentice-Hall, 1990. 370 p.

IBGE. Manual técnico da vegetação brasileira. Rio de Janeiro: Fundação Instituto Brasileiro de Geografia e Estatística, 1992. 92 p. (Série: Manuais técnicos em geociências n.1)

JARENKOW, J. A.; BAPTISTA, L. R. M. Composição florística e estrutura da mata com araucária na Estação Ecológica de Aracuri, Esmeralda, Rio Grande do Sul. Napaea, Porto
Alegre, v. 3, p. 9-18, 1987.

MARCHIORI, J. N. C. Dendrologia das gimnospermas, 2. ed. Santa Maria: UFSM, 2005. $161 \mathrm{p}$.

MULLER-DOMBOIS, D.; ELLENBERG, H. Aims and of vegetation ecology. New York: John Wiley \& Sons, 1974. 547 p.

NASCIMENTO, A. R. T. et al. Estrutura e padrões de distribuição espacial de espécies arbóreas em uma amostra de Floresta Ombrófila Mista em Nova Prata, RS. Ciência Florestal, Santa Maria, v. 11, n. 1, p. 105-119, 2001.

PEREIRA, C. A.; VIEIRA, I. C. G. A importância das florestas secundárias e os impactos de sua substituição por plantios mecanizados de grãos na Amazônia. Interciencia, Caracas, v. 26, p. $337-$ 341, 2001.

R DEVELOPMENT CORE TEAM. R: A language and environment for statistical computing. R Foundation for Statistical Computing, 2008. Disponível em: <(http://www.R-project.org) $>$ Acesso em: 18 de fevereiro de 2009.

SCHAAF, L. B. et al. Alteração na estrutura diamétrica de uma floresta ombrófila mista no período entre 1979 e 2000. Revista Árvore, Viçosa, v. 30, n. 2, p. 283-295, 2006.

SOLBRIG, O. T. Studies on the population biology of the genus Viola. II The effect of plant size on fiteness in Viola sororia. Evolution, Malden, v. 35, n. 6, p. 1080-1093, 1981.

SOUZA, V. C.; LORENZI, H. Botânica Sistemática: guia ilustrado pra identificação das famílias de Angiospermas da flora brasileira, baseado em APG II. Nova Odessa: Instituto Plantarum, 2005. 640 p. 